\title{
CONFIGURATIONAL STUDY ON THE ANTECEDENT CONDITIONS THAT AFFECT THE ADAPTATION TO A SPECIFIC CULTURAL CONTEXT USING THE QCA METHOD
}

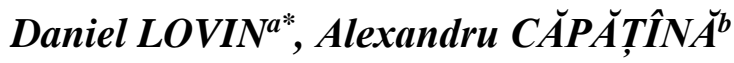 \\ a,b „Dunărea de Jos” University of Galați, Romania
}

\begin{abstract}
We live in an increasingly globalized world where intercultural interactions are increasingly present both in everyday life and within organizations. For many years, sport has become a symbol for what multiculturalism means. Sport is a global phenomenon, put into action by individuals from all over the world and watched everywhere. This article aims to address the issue of cultural intelligence in sport. Using the fsQCA method, this article shows the extent to which the four dimensions of cultural intelligence influence the adaptation of athletes to a new specific cultural context. Specifically, based on a questionnaire, it was analyzed how foreign athletes adapt in France and Romania. Although the work is done on a sample of athletes, the results could be extrapolated to other types of organizations.
\end{abstract}

KEYWORDS: adaptation, cultural intelligence, intercultural management, sport management

DOI: 10.24818/IMC/2021/04.04

\section{INTRODUCTION - THE QCA (QUALITATIVE COMPARATIVE ANALYSIS) METHOD}

One of the most important discoveries in the social sciences is the comparative qualitative analysis (Theim \& Dușa 2013). This assumes that a combination of antecedent conditions influences a certain phenomenon (Berg et al, 2008). A result can be determined by a causal configuration of conditions, which are all analyzed with the same rank of relevance (Ragin, 1987). Moreover, this method is increasingly used in various areas of activity where teamwork is crucial. This method began to be used in studies focused on the management of sports organizations. Thus, Kaufmann (2014) used the QCA method to find out which is the best solution for the pair of central defenders for the Swiss national football team. From the six players who could play in this position, the researchers chose two central defenders depending on the number of minutes played, the goals scored and the other partner in the center of the defense (Kaufmann, 2014). This method (QCA) is a research technique that eliminates the shortcomings of qualitative and quantitative research (Rihoux, Ragin, 2009). This method offers the possibility to analyze how several causal configurations influence an outcome (Ragin, 2014). Moreover, it shows the conditions that are sufficient (but not necessarily necessary) to reach a result. QCA reveals how causal configurations lead to a result (Woodside, 2010). The qualitative-comparative study brings to the fore the concept of equifinality, based on the idea that there may be a wide range of antecedent conditions leading to a certain desired result in a configurational research model (Fiss, 2007).

Human society does not change according to certain specific laws. People generally behave more or less chaotically, sometimes even against their own thoughts, beliefs or tastes. All human and social phenomena are characterized by a greater or lesser volume of chaotic action, which makes it impossible to measure and transpose them into mathematical laws (Duşa, 2014).

${ }^{*}$ Corresponding author. E-mail address: daniel.lovin@ugal.ro 
According to the principle of the excluded third party, a value is either necessary or not necessary. An item can be part of a crowd or it can be out of it. In 1987, Charles Ragin introduced the QCA in the social and political sciences. This paper is a basic paper in the field of qualitative-comparative research. Qualitative-comparative analysis can be applied at the level of macro-communities, the comparison of some individuals, but also of some micro-units of analysis, but it cannot provide results that can be generalized. The examples provided by vague sets vary between the macro and micro levels (Dușa, 2014).

There are situations in which the cases can only be distinct, of the yes or no type, where there is no middle ground. Even in these conditions, there are situations that are not as obvious, for example if a previous condition influences a variable. For example, one can analyze the factors that have an influence on adaptation to a new cultural context. In this paper, it is analyzed to what extent the metacognitive, cognitive, motivational and behavioral dimensions influence the adaptation to a new cultural context. However, it is not possible to say exactly which of these factors influences the adaptation to a cultural context, but there are certainly two limits.

For example, 0 (or $0 \%$ ) is the limit that indicates that a previous condition does not influence a result at all. At the opposite pole, 1 (or 100\%) means that a previous condition strongly influences a result. According to the same idea, if a previous condition is greater than 0.5 then it can be said that it influences the result. The closer the score is to 1 , the clearer it is that the causal condition influences the result. At the opposite pole, the closer the score is to 0.5 , the lower the previous condition has a weaker influence on the result, and the closer it gets to 0 , the weaker the influence. A perfect score equal to 0 or perfectly equal to 1 is not impossible, but it is quite rare. For example, a score of 0.12 does not automatically mean an exclusion from a crowd, but indicates that membership is rather weak, being more outside the crowd than inside it (Dușa, 2014).

Belonging to a class is a subject that is interpreted subjectively and so any statement in this regard is vague (fuzzy). For example, a person may be considered intelligent based on their coefficient, their ability to manage their emotions, their ability to solve problems, their ability to adapt to daily life, or their ability to adapt to a new cultural context. Appreciating an individual's intelligence is always a subjective assessment. Moreover, certain characteristics such as: intelligence, adaptability, communication skills or personality traits cannot be quantified in a direct way. Regardless of this subjectivity, it is important to explain in detail the scores obtained and to base the interpretations on solid theoretical frameworks.

Social human sciences are based primarily on inaccuracies and relative attributes. These relative attributes must be transposed into scores of one set or another. For example, a score above 0.7 of a previous condition could suggest a high influence on a variable. On the other hand, a score of 0.4 could be a weak influence, and a score below 0.2 could be an almost insignificant influence. However, it should be noted that sometimes language can be much more explicit through communication than through any numerical quantification.

Ragin (2000) indicates that fuzzy logic fits very well with the study of the social sciences, being a development of mathematics that combines extremely precise formal logic with verbal formulations, usually extremely imprecise. Vague sets can combine qualitative and quantitative attributes into a single instrument.

To exemplify operations with vague sets, Duşa (2014) uses the example of ethnic and racial affiliation in today's society, emphasizing that there are very few situations in which individuals are part of a single race. Nowadays, there are more and more individuals whose race is almost unidentifiable. Moreover, also regarding cultural diversity, Duşa (2014) states that a person belongs to a certain ethnic group if he feels, accepts and declares himself as belonging to that ethnic group. More important than anything else, what a person feels matters a lot, and feelings can be stronger or weaker, and this type of perception can only be measured through vague crowds. 
As for vague sets, there are several possible operations:

- Negation - which is the complement of a crowd and represents everything that does not belong to the crowd;

- Intersection - the situation in which in research there are cases that simultaneously meet several characteristics;

- Reunion - is the study of cases that have at least one feature;

- Concentration - sometimes some criteria can be so restrictive that none of the cases studied or known can reach high membership scores, so it can be said that the restrictions are relaxed so that those cases can fall into one of the categories.

\subsection{Calibration of vague sets}

The calibration of vague sets is done on a range between 0 and 1 , where 1 means the perfect membership, and 0 indicates the exclusion from a certain set. The closer it is to 1 , the higher the degree of belonging, while the closer it is to 0 , the lower the level of belonging. Calibration is considered practically an anchoring, a subjective division of results. For vague sets there are three standardization thresholds: full inclusion, complete exclusion and midpoint. This calibration process is a bridge between qualitative research and quantitative research as this anchoring is essentially a qualitative process, while the derivation of inclusion scores in the set is a quantitative process (Dușa, 2014).

Any numerical causal condition can be transformed into vague set scores, obtaining values between 0 and 1. Regardless of the unit of measurement of the initial numerical condition, vague scores can be obtained between 0 and 1, where 0 means that the initial values do not enter at all in that set, and 1 means that they are completely included in the set. Any values between 0 and 1 show how much or how little those values are included in the set.

For vague crowds a minimum of 3 thresholds is needed. This assignment is called direct assignment because all values that are outside the thresholds will be recoded either by 0 if they are lower than the exclusion threshold or by 1 if they are higher than the full inclusion threshold (Dușa, 2014).

\subsection{Necessity analysis}

Before analyzing the data using the truth table, the first step in qualitative-comparative analysis is to analyze the relationship of necessity between each of the causal condition (taken individually) and the phenomenon studied. In vague sets, whatever the combination of two fuzzy scores, it can be represented by coordinates in a Cartesian system, and that point can be positioned anywhere in this $\mathrm{xOy}$ plane. When the scores on the causal condition $\mathrm{x}$ are systematically higher than the scores on the studied phenomenon y, the points represented by these coordinates should be found in the triangle at the bottom right (below the main diagonal) of the Cartesian system xOy. The more included y is, the more necessary it turns out to be $\mathrm{x}$ for $\mathrm{y}$.

The QCA method has three main components: crisp QCA set (csQCA), multi-value QCA (mvQCA) and fuzzy QCA set (fsQCA). Case-based techniques, such as fsQCA and cluster analysis, have been used as a way to eliminate the limitations of variance-based statistical methods. These two techniques have similarities, as both use multidimensional spaces. A major difference between the two methods is the type of research questions they are able to address. More specifically, cluster analysis responds to challenges in which cases are more similar to each other, while fsQCA can identify the different causal configurations that constitute sufficient and / or necessary conditions for the expected outcome.

\section{RESEARCH FRAMEWORK}

The antecedent conditions used in this research involving the fsQCA method are the four components of cultural intelligence: metacognitive skills, cognitive skills, motivational skills and behavioral skills (Earley \& Ang, 2003), and the result is players' self-perception of adapting to a team abroad (Figure 1). 


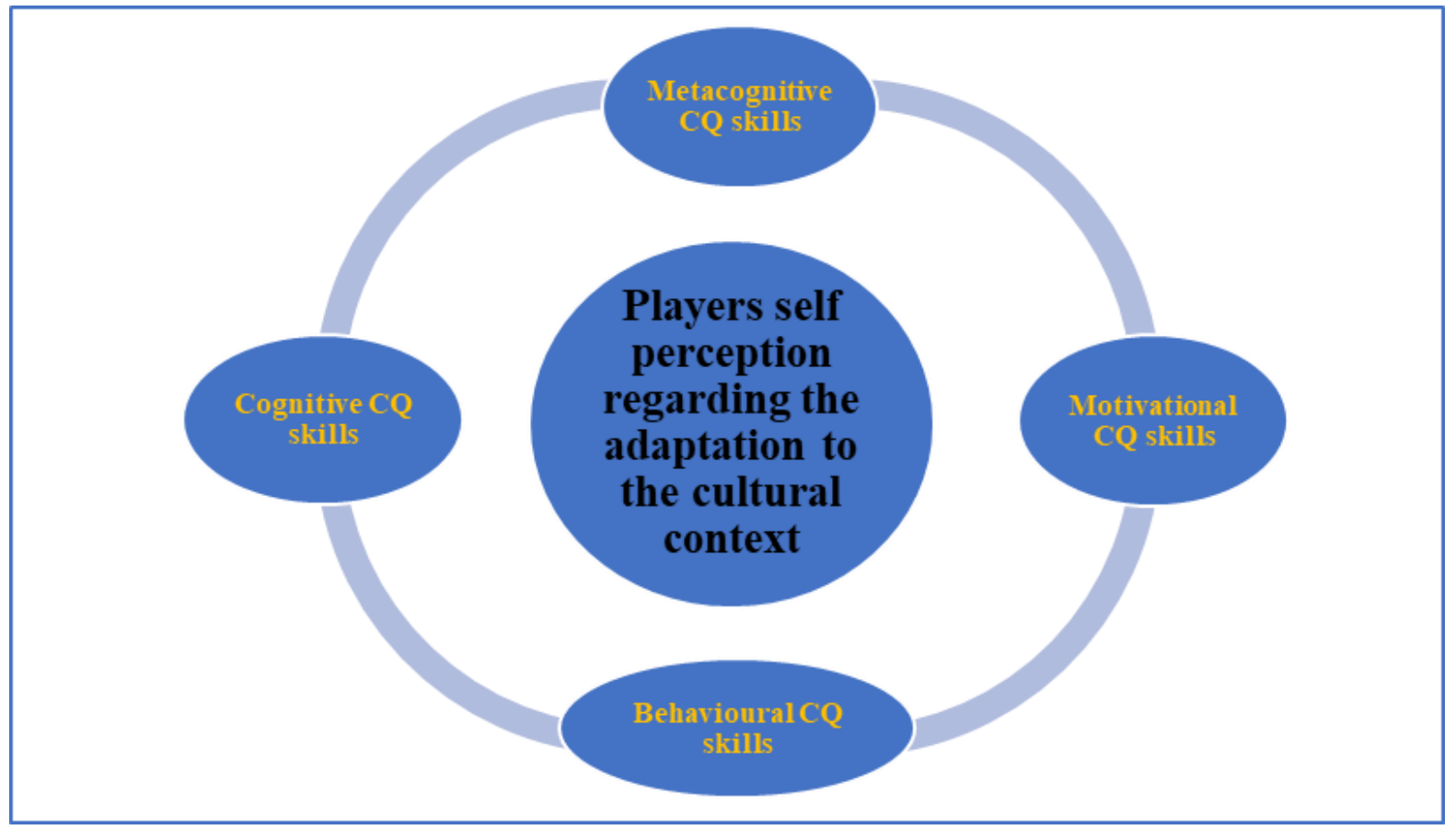

Figure 1. Conceptual model

Source: own contribution

\subsection{Data collection}

This study involves a comparative analysis of how foreign athletes adapt in two culturally different countries, namely Romania and France. Thus, through Google Forms, a questionnaire with 25 questions was created which was distributed to 150 foreign athletes evolving in France and Romania. All of these athletes practice team sports and the questionnaires were designed in English, French, Spanish and Portuguese to facilitate obtaining answers (https://forms.gle/Xkv92ft8vzApSPUw9). As in the case of the quantitative study, the items in the questionnaire were based on the works of Bucker (2015) and Ang (2003). Twenty of the 26 questions are about cultural intelligence skills, and 5 questions are about respondents. All items used a Likert scale from 1 (to a very small extent) to 5 (to a very large extent). Thus, 4 questions focused on the metacognitive dimension of cultural intelligence, 6 questions focused on the cognitive dimension of cultural intelligence, 5 questions focused on the motivational dimension of cultural intelligence and five focused on the behavioural dimension of cultural intelligence. In addition to all this, an item is formulated that aims at the selfperception of athletes on their own level of adaptation.

\subsection{Respondents}

To conduct this study, a questionnaire was prepared and distributed online to foreign athletes who play in multicultural teams in France and Romania. The responding athletes are both professional athletes and amateur athletes and practice various sports. In terms of gender repertoire, the number of men is higher than the number of women, but this disproportion can be explained by the fact that sport is more popular among men. Respondents are from all continents except Australia and Oceania and come from 49 countries.

\subsection{Calibration process}

Each antecedent condition of the conceptual model was evaluated by a 5-point scale: from Total Agreement to Total Disagreement (Table 1), while the result was evaluated by a distinct 5-point scale: from Full Adaptation to Very unadapted (Table 2). Fuzzy-sets QCA (fsQCA) reflects two types of configurations that include necessary and sufficient conditions. These configurations could be 
characterized by their presence or absence. The fsQCA method requires the definition of the result and the antecedent conditions. Both the causal conditions and the result must be calibrated in fuzzy sets with values between 0 and 1 . Specifically, the value 1 indicates full membership, while the value 0 does not indicate membership in the cluster. However, Woodside (2010) recommends avoiding assigning 0 to any established member and, consequently, we used 0.05 for this level.

Table 1. Calibration of the scales for the antecedent conditions

\begin{tabular}{|c|c|c|}
\hline Scale point & The fuzzy value & Membership \\
\hline Strongly agree/Very probably & 1 & Fully in \\
\hline Agree/Probably & 0.75 & More in than out \\
\hline $\begin{array}{c}\text { Neither agree or disagree/ } \\
\text { Possibly }\end{array}$ & 0.50 & More out than in \\
\hline Disagree/Probably not & 0.25 & Fully out \\
\hline $\begin{array}{c}\text { Strongly disagree/Definitely } \\
\text { not }\end{array}$ & 0.05 & (neither in nor out) \\
\hline
\end{tabular}

Source: adaptation after Ragin (2014)

Table 2. Calibration of result scales

\begin{tabular}{|c|c|c|}
\hline Scale point & The fuzzy value & Membership \\
\hline Complete adaptation & 1 & Fully in \\
\hline Partial adaptation & 0.75 & More in than out \\
\hline $\begin{array}{c}\text { Neither adapted nor } \\
\text { unadapted }\end{array}$ & 0.50 & More out than in \\
\hline Rather unadapted & 0.25 & Fully out \\
\hline Very unadapted & 0.05 & Cross-over (neither in nor out) \\
\hline
\end{tabular}

Source: adaptation after Ragin (2014)

The study, starting from the objective of the fsQCA logic, establishes three qualitative anchors for the calibration process of fuzzy sets: an anchor to define the complete membership, an anchor to define the non-complete membership and a midpoint (0.5).

\section{RESULT ANALYSIS}

\subsection{Analysis of research results for Romania}

The XOY plan specifies whether a certain condition is necessary to achieve the result, indicating how consistent a certain condition is. Consistency score is the extent to which a causal combination leads to a result. The coverage score represents how many cases with result are represented by a certain particular causal condition. The consistency score shows that the combination of the four dimensions of cultural intelligence (the metacognitive, cognitive, motivational and behavioral dimension) is sufficient to adapt to a new cultural context. The more cases in the OY plan, the more X proves to be more important for Y. In the case of foreign athletes evolving in Romania, it can be seen that most cases are in the OY plan. Of the 79 cases, 69 are in the OY plane, 6 cases are diagonal, and 4 are in the OX plane (figure 2).

Moreover, most of these cases have a degree of belonging to over 0.8. Thus, it can be stated that in the case of foreign athletes evolving in Romania, the combination of the four dimensions of cultural intelligence (metacognitive, cognitive, motivational and behavioral) influences the adaptation to a new cultural context. 


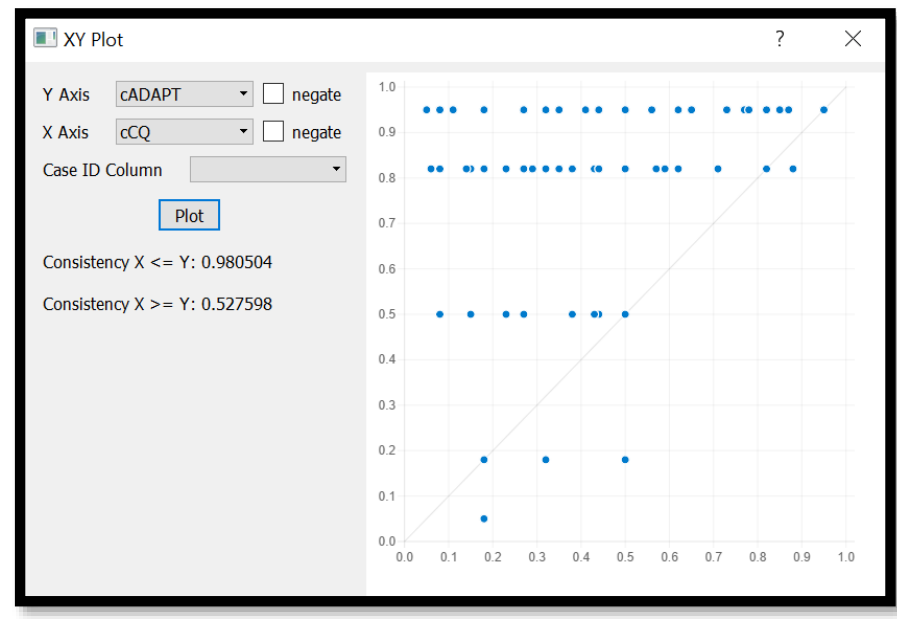

Figure 2. Distribution of cases in the XY plan chart for foreign athletes evolving in Romania Source: fsQCA output

The truth table (Table 3) highlights different case configurations. The analysis of the truth table serves to identify the causal patterns of sufficiency, as well as the combinations of conditions that are sufficient for the result. Each condition and result is represented in a column in the truth table. The additional columns show how many empirical cases show a certain configuration, if the cases are relevant to the impact of the result and what is the level of consistency of a causal configuration. Thus, seven configurations are distinguished in the research sample.

Table 3. Analysis of the truth table for foreign athletes evolving in Romania

\begin{tabular}{|c|c|c|c|c|c|c|c|c|}
\hline $\begin{array}{c}\mathrm{cMC} \\
\mathrm{Q}\end{array}$ & $\begin{array}{c}\mathrm{cCC} \\
\mathrm{Q}\end{array}$ & $\begin{array}{c}\mathrm{cMOTC} \\
\mathrm{Q}\end{array}$ & $\begin{array}{c}\mathrm{cBC} \\
\mathrm{Q}\end{array}$ & $\begin{array}{c}\text { numbe } \\
\mathrm{r}\end{array}$ & $\begin{array}{c}\mathrm{cADAP} \\
\mathrm{T}\end{array}$ & $\begin{array}{c}\text { Raw } \\
\text { consistenc } \\
\mathrm{y}\end{array}$ & $\begin{array}{c}\text { Proportional } \\
\text { reduction of } \\
\text { inconsistenc } \\
\mathrm{y}\end{array}$ & $\begin{array}{c}\text { Symmetrica } \\
\text { consistency }\end{array}$ \\
\hline 1 & 1 & 1 & 1 & 22 & 1 & 0.9805 & 0.9702 & 0.9729 \\
\hline 0 & 0 & 1 & 1 & 2 & 1 & 0.9669 & 0.9105 & 0.9105 \\
\hline 1 & 1 & 1 & 0 & 4 & 1 & 0.9636 & 0.9232 & 0.9551 \\
\hline 1 & 0 & 1 & 0 & 13 & 1 & 0.9597 & 0.9237 & 0.9237 \\
\hline 1 & 0 & 1 & 1 & 12 & 1 & 0.9509 & 0.9134 & 0.9186 \\
\hline 0 & 0 & 0 & 0 & 1 & 1 & 0.9491 & 0.7758 & 0.8288 \\
\hline 0 & 0 & 1 & 0 & 2 & 1 & 0.9432 & 0.8397 & 0.8702 \\
\hline
\end{tabular}

Source: fsQCA software output

The Quine-McCluskey algorithm provides complex solutions that reveal valuable information about combinations of antecedent conditions that influence the outcome. All these combinations (presented in Table 4) are successful methods for athletes who want to adapt to a new cultural context.

The first solution offered by the Quine-McCluskey algorithm suggests a low level of cognitive skills of cultural intelligence, combined with strong motivational skills gives a consistency of 0.9185. Even if it is a low level of cognitive skills, it can be said that motivation can compensate for cognitive skills. 
Table 4. The complex solution offered by the Quine-McCluskey algorithm for the Romanian sample

\begin{tabular}{|c|c|c|c|}
\hline Complex solution & Raw coverage & Unique coverage & Consistency \\
\hline$\sim \mathrm{cCCQ}^{*} \mathrm{cMOTCQ}$ & 0.5548 & 0.0237 & 0.9185 \\
\hline $\mathrm{cMCQ}^{*} \mathrm{cMOTCQ}$ & 0.8224 & 0.2985 & 0.9367 \\
\hline$\sim \mathrm{cMCQ}^{*} \sim \mathrm{cCCQ}{ }^{*} \sim \mathrm{cBCQ}$ & 0.2609 & 0.0033 & 0.9319 \\
\hline Solution coverage: 0.8568 & \multicolumn{4}{l}{} \\
\hline Solution consistency: 0.9188
\end{tabular}

Source: fsQCA software output

Cognitive skills refer to an individual's ability to know a foreign language, to know the economic and legal system of another culture or to know the cultural and religious values of another culture. However, all these aspects cannot be assimilated without a strong motivation. Motivation is what gives energy to the individual and mobilizes him to carry out a certain activity.

The second solution proposed by the Quine-McCluskey algorithm is a combination of a high level of metacognitive skills and a high level of motivational skills. And in this case the motivational dimension is present, only that it is in combination with the metacognitive dimension of cultural intelligence. The metacognitive dimension requires superior thinking skills. It involves asking questions and looking for answers to certain problems and situations. Thus, this second solution, which presents the combination of a high level of cognitive skills and motivational skills, we obtained a consistency of 0.9367 .

Last but not least, the third solution proposed by the Quine - McCluskey algorithm involves a combination of a low level of metacognitive skills, a low level of cognitive skills and a low level of behavioural skills. Of all the three software solutions proposed, it is the only one that contains three dimensions of cultural intelligence. This combination of these three dimensions has a consistency of 0.9319. This solution suggests that in order to adapt to a new cultural context, it is necessary to have cognitive skills, but also behavioural skills.

In the Romanian sample, the principle of equifinality is fulfilled because 3 causal configurations lead to a certain result. In addition, the fsQCA software allows testing the conditions necessary to achieve a certain result (Table 5). Thus, the same four causal combinations were tested for both the Romanian sample and the French sample.

Table 5. Necessary conditions for fulfilling the result in Romania

\begin{tabular}{|c|c|c|}
\hline Tested conditions & Consistency & Coverage \\
\hline cMCQ+cCCQ+cMOTCQ+cBCQ & 0.9619 & 0.8948 \\
\hline cCCQ+cBCQ+cMOTCQ & 0.9349 & 0.8982 \\
\hline cMCQ+cBCQ+cMOTCQ & 0.9617 & 0.8971 \\
\hline cBCQ+cMOTCQ & 0.9312 & 0.9021 \\
\hline
\end{tabular}

Source: output fsQCA

For the sample from Romania, the combination with the highest consistency (0.9619) is represented by the combination of all four causal conditions. Thus, it can be said that in order to fulfil the adaptation to a new cultural context, it is necessary for an athlete to possess all four dimensions of cultural intelligence. The combination with the second highest consistency (0.9617) is the combination of three causal conditions, namely the metacognitive dimension, the behavioural 
dimension and the motivational dimension of cultural intelligence. Of these combinations, only the cognitive dimension is missing. At the same time, it must be taken into account that metacognitive skills are considered to be superior to cognitive abilities, which suggests that their possession helps to a greater extent in the process of adapting to a new culture.

Thus, it is explained why the third combination of causal conditions represented by the combination of cognitive dimension, behavioural dimension and motivational dimension has a consistency of 0.9349 , lower than the consistency of the previous combination in which the metacognitive dimension is at the expense of cognitive dimension. The last causal condition tested is the combination of only two dimensions of cultural intelligence, namely the behavioural dimension and the motivational dimension. This last combination tested, although represented by only two elements, has a consistency of 0.9312 and suggests that in order to adapt to a new culture it may be necessary to master only these two dimensions. In this situation we can specify the special importance of the motivational dimension in the adaptation process, but also the fact that the behavioural dimension acts as a result of the influence of cognition. At the same time, it should be noted that in all four necessary conditions tested are present both the motivational dimension and the behavioural dimension.

\subsection{Analysis of research results for France}

As in the case of foreign athletes evolving in Romania and in the case of foreign athletes evolving in France, it can be seen that most cases are in the OY plan. In the case of foreign athletes evolving in France, there are only 5 cases in the OX plane and no case that is diagonal (Figure 3 ).

In addition, as in the case of Romania, most of the cases have a degree of affiliation of over 0.8 . The lower number of cases below the diagonal can be explained by the fact that many of the foreign athletes evolving in France come from French-speaking countries and the athletes already speak French. In these cases, adaptation should be facilitated by the fact that mastering the French language makes communication easier and the adaptation process can be facilitated.

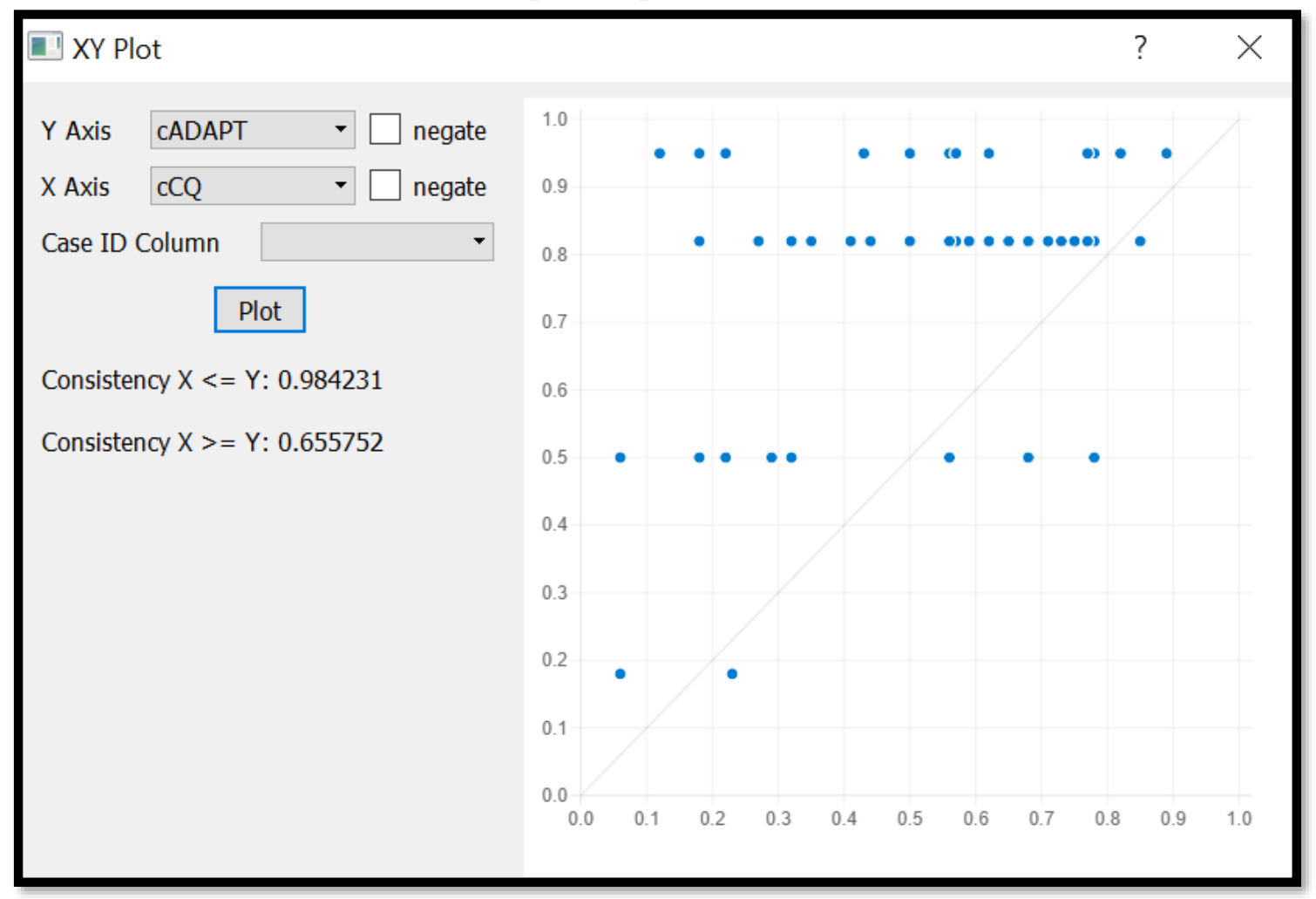

Figure 3. Distribution of cases in the XY plan chart for foreign athletes evolving in France Source: fsQCA output 
In the case of the sample of athletes from France, there are 6 configurations in the truth table (Table 6).

Table 6. Analysis of the truth table for foreign athletes evolving in France

\begin{tabular}{|l|l|l|l|l|l|l|l|l|}
\hline cMCQ & cCCQ & cMOTCQ & cBCQ & number & cADAPT & $\begin{array}{l}\text { Raw } \\
\text { consistency }\end{array}$ & $\begin{array}{l}\text { Proportional } \\
\text { reduction of } \\
\text { inconsistency }\end{array}$ & $\begin{array}{l}\text { Symmetrical } \\
\text { consistency }\end{array}$ \\
\hline 0 & 0 & 1 & 1 & 2 & 1 & 0.9967 & 0.9913 & 0.9913 \\
\hline 0 & 0 & 1 & 0 & 1 & 1 & 0.9856 & 0.9548 & 0.9746 \\
\hline 1 & 1 & 1 & 1 & 40 & 1 & 0.9842 & 0.9777 & 0.9981 \\
\hline 1 & 0 & 1 & 1 & 7 & 1 & 0.9828 & 0.9660 & 0.9953 \\
\hline 1 & 0 & 0 & 0 & 1 & 1 & 0.9672 & 0.9096 & 0.9096 \\
\hline 0 & 0 & 0 & 0 & 1 & 1 & 0.9334 & 0.7892 & 0.7892 \\
\hline
\end{tabular}

If in the Romanian sample two solutions proposed by the Quine-McCluskey algorithm represent a combination of only two dimensions of cultural intelligence, in the case of the French sample all the proposed solutions contain three dimensions of cultural intelligence (Table 7).

Table 7. The complex solution offered by the Quine-McCluskey algorithm for the French sample

\begin{tabular}{|c|c|c|c|}
\hline Complex solution & Raw coverage & Unique coverage & Consistency \\
\hline$\sim \mathrm{cCQ}^{*} \sim \mathrm{cMOTCQ}^{*} \sim \mathrm{cBCQ}$ & 0.2444 & 0.0162 & 0.9208 \\
\hline$\sim \mathrm{cMCQ}^{*} \sim \mathrm{cCCQ}{ }^{*} \mathrm{cMOTCQ}$ & 0.2806 & 0.0231 & 0.9876 \\
\hline $\mathrm{cMCQ}^{*} \mathrm{cMOTCQ}{ }^{*} \mathrm{cBCQ}$ & 0.7517 & 0.4797 & 0.9664 \\
\hline Solution coverage: 0.8033 & \multicolumn{4}{l}{} \\
\hline Solution consistency: 0.9438
\end{tabular}

\section{Source:output fsQCA}

The first solution offered by the Quine-McCluskey algorithm for the French sample is a low combination of the cognitive dimension, the motivational dimension and the behavioral dimension of cultural intelligence and has a consistency of 0.9208. This first solution suggests that an even low combination of these three factors may be a solution for adapting athletes to a new cultural context. The second complex solution is represented by a low combination between metacognitive and cognitive dimensions and a high combination of motivational dimension, having at the same time the highest consistency (0.9876). It reiterates the importance of motivation in the adaptation process. A high level of motivation combined with cognitive and metacognitive skills is a very good solution for adapting athletes to a new cultural context. Thus, it is important to note that those who recruit international athletes should assess their motivation and desire to adapt to a new culture.

Last but not least, the last complex solution proposed is a combination of a high level of metacognitive skills, a high level of motivational skills and a high level of behavioral skills. This mix of skills has a high consistency of 0.9664 and is a good solution for adapting athletes to a new cultural context.

In the French sample, the principle of equifinality is fulfilled as 3 causal configurations lead to a certain result. Regarding the analysis of the conditions required for the sample in France, the four conditions tested are ranked identically, except that a difference is noted in terms of consistency (Table 8). 
"Managing People and Organizations in a Global Crisis" $4^{\text {th }}-5^{\text {th }}$ November, 2021, BUCHAREST, ROMANIA

Table 8. Requirements for obtaining the result in France

\begin{tabular}{|c|c|c|}
\hline Tested conditions & Consistency & Coverage \\
\hline cMCQ+cCCQ+cMOTCQ+cBCQ & 0.9462 & 0.9237 \\
\hline cCCQ+cBCQ+cMOTCQ & 0.9163 & 0.9371 \\
\hline cMCQ+cBCQ+cMOTCQ & 0.9439 & 0.9239 \\
\hline cBCQ+cMOTCQ & 0.9059 & 0.9368 \\
\hline
\end{tabular}

Source: output fsQCA

For the French sample the consistency of the tested conditions is slightly lower, but even so, all tested conditions have a consistency of over 0.90 which suggests that all these conditions can be successfully applied to adapt to a new cultural context. Conversely, even if the conditions tested have a lower consistency, in terms of coverage it is higher for the sample from France.

\section{CONCLUSIONS AND DISCUSSIONS}

In the age we live in, the world is intercultural and many sports organizations have become a global business. Managers, staff, and players have to be aware to cultural differences and put intercultural skills into practice. Currently, athletes have the opportunity to play abroad, in a country where the culture is different from the culture in which they were raised. Regardless of the country, region, club or sports level, the culture in the general sense and the organizational culture of that club will influence the adaptation of athletes and managers and implicitly their efficiency on the field. It is crucial for stakeholders to understand the impact of cultural differences. Managers and football players need to put into practice their intercultural skills and to behave appropriately in the new culture.

The QCA method, on the basis of which this chapter was made, highlights aspects of special importance for the intercultural management applied in sports organizations. First, it should be noted that the present study confirms that the four causal conditions (metacognitive dimension, cognitive dimension, motivational dimension and behavioural dimension of cultural intelligence) influence the outcome, namely players' self-perception of adaptation to a new cultural context. Basically, this study highlights the role of all four dimensions of cultural intelligence in adapting to a new cultural context, but unlike previous studies, it was conducted on a sample of foreign athletes evolving in France and Romania. Moreover, the vast majority of responding athletes have a rich multicultural experience, which adds value to this study. Secondly, another particularly important aspect to be mentioned is the fact that analyzing the solutions proposed by the fsQCA software, it is noted that motivation plays a particularly important role in the adaptation process. Thus, decision-makers who select international athletes should pay more attention to this issue and seek to recruit athletes with a high level of motivation. As far as athletes are concerned, they should analyze their level of motivation and always be motivated to overcome the obstacles of adapting to a new cultural context.

Cultural differences do not only appear in environments such as industrial, but in such situations the lack of adaptation is more visible. Lately, the issue of cultural maladaptation of athletes has begun to be brought to light more and more. Sport has become a business and the decision makers involved in the phenomenon are trying to make a profit by any means. In sports, there are many more elements that influence the performance of athletes, and many individuals involved in the phenomenon think that cultural differences are not so important and thus do not give more importance. If in the industrial sector, performance is affected by cultural differences, it is clear that this can also happen in sports. 
There are many situations in which athletes or managers have succeeded successfully in a cultural context, but the high has failed miserably.

\section{REFERENCES}

Berg S., Dirk, G., Rihoux, B., \& Ragin, C. (2008) - Qualitative Comparative Analysis (QCA) as an Approach In Configurational comparative methods. Qualitative Comparative Analysis (QCA) and related techniques, eds. RIHOUX, Benoit and Charles Ragin. Thousand Oaks and London: Sage, 1-18.

Bücker, J., Furrer, O., \& Lin, Y. (2015). Measuring cultural intelligence (CQ) A new test of the CQ scale. International Journal of Cross Cultural Management, 15(3), 259-284.

Dușa, A. (2014). Elemente de analiză comparativă. Editura Tritonic, București.

Earley, P. C., \& Ang, S. (2003). Cultural intelligence: Individual interactions across cultures.

Fiss, P. C. (2007). A set-theoretic approach to organizational configurations. Academy of management review, 32(4), 1180-1198.

Kaufmann, D. (2014). Assessing collective defensive performances in football: A Qualitative Comparative Analysis of central back pairs.

Ragin, C. (1987). The Comparative Method: Moving Beyond Qualitative and Quantitative Strategies. Berkeley and Los Angeles: University of California Press.

Ragin, C. (2000). Fuzzy-set social science. Chicago: The University of Chicago Press.

Ragin, C. C. (2014). The comparative method: Moving beyond qualitative and quantitative strategies. Berkely and Los Angeles, USA: University of California Press.

Rihoux, B., \& Ragin, C. C. (2009). Configurational comparative methods: Qualitative comparative analysis (QCA) and related techniques. Thousand Oaks, CA: Sage.

Thiem, A., \& Duşa, A. (2013) - Boolean Minimization in Social Science Research: A Review of Current Software for Qualitative Comparative Analysis (QCA). Social Science Computer Review 31(4), 505-521.

Woodside, A. G. (2010). Case study research: Theory, methods and practice: Theory, methods, practice. Bingley, UK: Emerald Group Publishing.

\section{Appendix}

\section{Questionnaire used for data collection}

\begin{tabular}{|c|c|}
\hline \multirow{4}{*}{$\begin{array}{l}\text { Metacognitive CQ } \\
\text { skills }\end{array}$} & $\begin{array}{l}\text { 1. I am conscious of the cultural knowledge I use when interacting } \\
\text { with people with different cultural backgrounds }\end{array}$ \\
\hline & $\begin{array}{l}\text { 2. I adjust my cultural knowledge as I interact with people from a } \\
\text { culture that is unfamiliar to me. }\end{array}$ \\
\hline & $\begin{array}{l}\text { 3. I am conscious of the cultural knowledge I apply to cross- } \\
\text { cultural interactions. }\end{array}$ \\
\hline & $\begin{array}{l}\text { 4. I check the accuracy of my cultural knowledge as I interact with } \\
\text { people from different cultures. }\end{array}$ \\
\hline \multirow{6}{*}{ Cognitive CQ skills } & 5. I know the legal and economic systems of other cultures. \\
\hline & 6. I know the rules (e.g., vocabulary, grammar) of other languages. \\
\hline & 7. I know the cultural values and religious beliefs of other cultures. \\
\hline & 8. I know the marriage systems of other cultures. \\
\hline & 9. I know the arts and crafts of other cultures. \\
\hline & $\begin{array}{c}\text { 10. I know the rules for expressing non-verbal behaviors in other } \\
\text { cultures. }\end{array}$ \\
\hline
\end{tabular}




\begin{tabular}{|c|c|}
\hline \multirow{5}{*}{$\begin{array}{c}\text { Motivational CQ } \\
\text { skills }\end{array}$} & 11. I enjoy interacting with people from different cultures. \\
\hline & $\begin{array}{l}\text { 12. I am confident that I can socialize with locals in a culture that } \\
\text { is unfamiliar to me. }\end{array}$ \\
\hline & $\begin{array}{l}\text { 13. I am sure I can deal with the stresses of adjusting to a culture } \\
\text { that is new to me. }\end{array}$ \\
\hline & 14. I enjoy living in cultures that are unfamiliar to me. \\
\hline & $\begin{array}{l}\text { 15. I am confident that I can get accustomed to the shopping } \\
\text { conditions in a different culture. }\end{array}$ \\
\hline \multirow{5}{*}{$\begin{array}{l}\text { Behavioural CQ } \\
\text { skills }\end{array}$} & $\begin{array}{l}\text { 16. I change my verbal behavior (e.g., accent, tone) when a cross } \\
\text { cultural interaction requires it. }\end{array}$ \\
\hline & $\begin{array}{l}\text { 17. I use pause and silence differently to suit different } \\
\text { crosscultural situations. }\end{array}$ \\
\hline & $\begin{array}{l}\text { 18. I vary the rate of my speaking when a cross-cultural situation } \\
\text { requires it. }\end{array}$ \\
\hline & $\begin{array}{l}\text { 19. I change my non-verbal behavior when a cross-cultural } \\
\text { interaction requires it. }\end{array}$ \\
\hline & $\begin{array}{l}\text { 20. I alter my facial expressions when a cross-cultural interaction } \\
\text { requires it. }\end{array}$ \\
\hline $\begin{array}{c}\text { Result } \\
\text { Athletes' self- } \\
\text { perception of } \\
\text { cultural adaptation }\end{array}$ & 21. I feel culturally adapted to the club where I am playing now \\
\hline
\end{tabular}

Source: Bücker, J., Furrer, O., \& Lin, Y. (2015) 